\title{
EVALUATION OF THE EFFECTIVENESS AND RELIABILITY OF LOW-DOSE TRANEXAMIC ACID USED IN ADOLESCENT IDIOPATHIC SCOLIOSIS SURGERY
}

\author{
๑ Çağla Bali' ๑ Ümit Özgür Güler²
}

\author{
${ }^{1}$ Başkent University Faculty of Medicine, Adana Dr. Turgut Noyan Application and Research Center, Department of Anesthesiology and \\ Reanimation, Adana, Turkey \\ ${ }^{2}$ Başkent University Faculty of Medicine, Adana Dr. Turgut Noyan Application and Research Center, Department of Orthopedics and \\ Traumatology, Adana, Turkey
}

\begin{abstract}
Objective: Tranexamic acid (TXA) is an antifibrinolytic drug that is frequently used in pediatric spinal fusion surgery to prevent bleeding. In recent publications, TXA was used in very high doses, but there is no consensus about the dosage of TXA in adolescent idiopathic scoliosis (AIS) surgeries. Our aim was to investigate the effectiveness and safety of a low-dose regimen on perioperative bleeding in AIS surgery.

Materials and Methods: A total of 25 patients diagnosed as having AIS were reviewed retrospectively. We retrieved demographic and intraoperative data, and bleeding related outcomes of the patients from their medical records. The primary outcomes were estimated blood loss and the transfusion requirements.

Results: Twenty-two (88\%) of the patients were women. The mean age was 13.72 years. The mean number of vertebrae fused was 11.79. Estimated blood loss was $616 \mathrm{~mL}$, and intraoperative mean red blood cell unit transfusion was determined as 0.8 units. No adverse event was observed related to TXA usage.

Conclusion: The use of low-dose TXA is a reliable and efficient method to reduce bleeding in AIS surgery.

Keywords: Tranexamic acid, adolescent idiopathic scoliosis, blood loss
\end{abstract}

\section{INTRODUCTION}

Adolescent idiopathic scoliosis (AIS) is a three-dimensional spinal deformity involving the sagittal, horizontal, and frontal planes, and affecting $1-3 \%$ of the child population aged $10-$ 16 years. Although the pathogenesis is not clearly explained, hereditary factors are in the foreground ${ }^{(1)}$. Posterior spinal fusion (PSF) surgery is the preferred type of surgery in these patients, but long surgical time and extensive dissections can cause significant blood loss. In addition, multiple osteotomies and wide muscle dissections performed during surgery, preoperative high Cobb angle, and increased number of vertebrae requiring fusion, cause an increase in the amount of bleeding ${ }^{(2)}$. Due to allogeneic blood transfusion performed after severe bleeding; transfusion reactions and blood-borne pathogen transmission increase the risk of morbidity and mortality ${ }^{(3)}$. Therefore, besides techniques such as hypotensive anesthesia, acute normovolemic hemodilution, preoperative autologous donation, there are also pharmacological agents used to prevent bleeding ${ }^{(4,5)}$.

Tranexamic acid (TXA) is an antifibrinolytic drug that is a synthetic lysine analog. It prevents fibrin degradation by preventing the conversion of plasminogen to plasmin ${ }^{(6)}$. TXA is frequently used in major surgery with bleeding, and its effectiveness in pediatric spinal fusion surgery has been reported many times in the literature ${ }^{(7-9)}$.

Although TXA is used in different doses in the literature, we have been using it in our clinic, in the surgery of AIS cases with a loading dose of $10 \mathrm{mg} / \mathrm{kg}$ and a maintenance dose of $1 \mathrm{mg} / \mathrm{kg} / \mathrm{h}$ since 2015. In this retrospective study, our aim is to investigate the effect and safety of this low-dose regimen on perioperative bleeding applied in AIS patients with PSF between 2015-2020. 


\section{MATERIALS AND METHODS}

This retrospective study was approved by Başkent University Instutional Review Board (KA21/29). After the approval of the ethics committee, 25 consecutive patients with American Society of Anesthesiologists (ASA) risk classification I-III, between the ages of 12-15, who underwent PSF surgery with spinal cord monitoring, in our hospital between the years 2015-2020, were evaluated. Patients with a diagnosis of neuromuscular or congenital scoliosis, patients who underwent anterior revision, or lengthening spinal surgery, and patients with incomplete data were excluded from the study. The patients' information was obtained from archived files, anesthesia records, and the medical data system used in our hospital.

Patients' age, gender, weight, height, body mass index, preoperative hemoglobin levels, and preoperative Cobb angle were obtained from pre-anesthesia records. From the intraoperative records, operation time, fusion levels, estimated blood loss (EBL), and the amount of erythrocyte used [red blood cell unit (RBC)] were obtained. Then postoperative hemoglobin value, amount of RBC used, postoperative Cobb angle, hospital stay, and complications were obtained from the postoperative records.

\section{Anesthesia Management}

All patients were sedated with $0.05 \mathrm{mg} / \mathrm{kg}$ midazolam (Dormicum ${ }^{\oplus} 5 \mathrm{mg} / 5 \mathrm{~mL}$ ampoule, Deva, Istanbul/Turkey) 15 minutes before the operation, and taken to the operating room. In anesthesia management, after standard anesthesia monitoring, anesthesia induction was applied with Propofol (2-3 mg/kg, Propofol ${ }^{\ominus} 1 \% 200 \mathrm{mg} / 20 \mathrm{~mL}$ ampoule, Fresenius Kabi, Austria), Fentanyl (2 $\mu \mathrm{g} / \mathrm{kg}$ Fentanyl $^{\oplus} 0,05 \mathrm{mg} / \mathrm{mL}$ ampoule, Johnson\&Johnson, Istanbul/Turkey) and Rocuronium Bromide $\left(0.3 \mathrm{mg} / \mathrm{kg}\right.$ Esmeron ${ }^{\oplus} 50 \mathrm{mg} / 5 \mathrm{~mL}$ ampoule, Merck Sharp Dohme, Istanbul/Turkey). After intubation, invasive arterial catheterization from the radial artery, and central venous catheterization from the right internal jugular vein, were performed. Additionally, an oral temperature probe, bladder catheterization, and 2 peripheral vascular access were provided. After the patients were positioned properly in the prone position, baseline electrophysiological values were obtained for neurophysiological monitoring. For the maintenance of anesthesia, $50 \%$ nitric oxide, $50 \%$ oxygen, and total intravenous anesthesia (propofol-remifentanil infusion; 4-6 mg/kg/st - 1-2 $\mu \mathrm{g} \mathrm{kg} / \mathrm{h}$ ) was applied. To reduce blood loss, controlled hypotension was applied with an average arterial pressure of $60-65 \mathrm{mmHg}$. Acid-base balance and hemoglobin levels were monitored with periodic blood gas sampling. During the anesthesia, besides hemodynamic data and bleeding, temperature and external warming were performed. Neurophysiological monitoring was performed with all patients to monitor all stages of surgery. TXA (Transamine $50 \mathrm{mg} /$ $\mathrm{mL}$ ampoule, Istanbul/Turkey) was administered immediately after the induction of anesthesia at a dose of $10 \mathrm{mg} / \mathrm{kg}$ in
$100 \mathrm{~mL}$ isotonic for 15 minutes. Its maintenance dose was started before the skin incision as $1 \mathrm{mg} / \mathrm{kg} / \mathrm{h}$, and continued intraoperatively. It was stopped at the end of the surgery. The amount of intraoperative bleeding was determined by the padsponge count and the amount of blood accumulated in the suction tanks. The irrigation fluid and the amount of blood in the collection jars were carefully calculated by the nurse. The amount of blood in the pads and sponges was determined by making an estimated calculation according to the blood saturation. RBC suspension was given when the hemoglobin level was $9 \mathrm{gr} \mathrm{dL}^{-1}$ in blood gas monitoring. During the closure phase of the surgery, $0.15 \mathrm{mg} / \mathrm{kg}$ intravenous morphine and $15 \mathrm{mg} / \mathrm{kg}$ paracetamol were applied to all patients. Patients without hemodynamic problems and acid-base imbalances were extubated and taken to the recovery room. Patients who started morphine administration with a patient-controlled analgesia device, and whose pain control was provided, were sent to the orthopedics service for postoperative follow-up. Patients with an estimated amount of bleeding more than $30 \%$ of their total blood volume, those who developed metabolic or respiratory acidosis, those who were hemodynamically unstable, and those who received inotrope/vasopressor support were referred to the intensive care unit for a closer postoperative follow-up.

\section{Surgical Technique}

Posterior surgery was performed in all patients in the prone position. The skin and subcutaneous tissue were passed through a midline incision. The paraspinal muscles were stripped subperiosteally then polyaxial pedicle screws were placed at specified levels using the appropriate technique. The curvatures were corrected by derotation, compression, and distraction maneuvers over the rod. After the instrumentation was completed, the posterior elements were decorticated, and bony areas for fusion were created using allografts. Additional osteotomies were not performed for any patient. Following bleeding control and wound washing, vancomycin powder was applied over the implants and the surgical site. Postop wound drainage was followed by the hemovac drainage system. The hemovac drain was withdrawn on the first postoperative day and the patients were mobilized.

\section{Statistical Analysis}

The analysis of the data was performed using the Statistical Package for the Social Sciences (SPSS) for Windows 21 (Chicago IL., USA) package program. For continuous variables, descriptive statistics are used as the mean and standard deviation, or minimum-maximum, and the categorical variables were shown as the number of cases and percentage (\%).

\section{RESULTS}

Twenty-five consecutive patients who were operated on, in our hospital, with a diagnosis of AIS were included in the study. The mean age of the patients was 13.72 (minimum-maximum: $13-15)$ and 22 (88\%) of them were women. In the preoperative 
anesthesia evaluation of patients, $72 \%$ ASA I, 24\% ASA II, of the ASA II patients $(n=6) ; 4$ had mild asthma, 1 had diabetes mellitus, and 1 had epilepsy. One patient with ASA III had a diagnosis of 2/4 mitral insufficiency. The average weight of the patients was $40.8 \mathrm{~kg}(32-58)$ and their average height was 151 $\mathrm{cm}$ (140-176). The mean preoperative hb value of all cohort was $13.3 \mathrm{~g} \mathrm{dL}^{-1}$ (11.7-16.9), and the preoperative Cobb angles averaged $51^{\circ}\left(40-65^{\circ}\right)$ (Table 1$)$.

Patients were instrumented between T1-L5 levels, and the mean number of vertebrae fused was 11.79 (7-16). The mean operation time was 213 minutes (120-300 $\mathrm{min}$ ), and the average hospital stay of the whole patient group was 5.48 days (3-8). Considering the complications of the whole study group, instrumentation was extended from T10 to T2 due to increased thoracic curvature after 2 years in one patient, and debridement was applied 2 days after surgery due to early wound infection in another patient. However, no complications related to the use of TXA were observed (Table 2).

Considering the results related to bleeding, the mean EBL of the whole study group was $616 \mathrm{~mL}$ (250-1100), and intraoperative mean RBC transfusion was determined as 0.8 units $(0-2)$. When the blood transfusions given to the patients during surgery were examined, it was determined that 9 (36\%) patients did

Table 1. Mean demographics of the patients

\begin{tabular}{|c|c|}
\hline Age (y) & $13.72 \pm 0.79$ \\
\hline Gender (Male/female) n & $3 / 22$ \\
\hline ASA physical status (I/II/III) n & $18 / 6 / 1$ \\
\hline Weight (kg) & $40.8 \pm 7.74$ \\
\hline Height (cm) & $151 \pm 7.71$ \\
\hline BMI & $17.7 \pm 2.49$ \\
\hline Preoperative $\mathrm{hb}\left(\mathrm{g} \mathrm{dL}^{-1}\right)$ & $13.3 \pm 1.02$ \\
\hline Preoperative Cobb angle $\left({ }^{\circ}\right)$ & $51.0 \pm 8.33$ \\
\hline \multicolumn{2}{|c|}{$\begin{array}{l}\text { Data is given as numbers for gender and ASA, otherwise mean } \pm \\
\text { standard deviation. } \\
\mathrm{n} \text { : Number, ASA: American Society of Anesthesiologists, BMI: Body mass } \\
\text { index }\end{array}$} \\
\hline
\end{tabular}

Table 2. Operation related factors, bleeding related outcomes, hospitalization time and complications

\begin{tabular}{|ll|}
\hline Postoperative Cobb angle $\left(^{\circ}\right)$ & $8.36 \pm 6.70$ \\
\hline Number of levels fused & $11.79 \pm 2.93$ \\
\hline Operation time (minutes) & $213.60 \pm 54.61$ \\
\hline Estimated blood loss (mL) & $616 \pm 228.54$ \\
\hline Intraoperative transfusion (unit) & $0.80 \pm 0.70$ \\
\hline Postoperative transfusion (unit) & $0.56 \pm 0.65$ \\
\hline Total transfusion (unit) & $1.36 \pm 1.03$ \\
\hline Postoperative hb (g dL ${ }^{-1}$ ) & $11.24 \pm 1.16$ \\
\hline Hospitalization time (days) & $5.48 \pm 1.04$ \\
\hline Complications (n) & 2 \\
\hline
\end{tabular}

Data is given as numbers for complications otherwise mean \pm standard deviation not receive a transfusion, 12 (48\%) patients received 1 unit and $4(16 \%)$ patients received 2 units of red blood cells. In the postoperative period, mean RBC transfusion was determined as 0.5 units $(0-2)$. In this period, $13(52 \%)$ patients had no transfusion, while 10 (40\%) patients received 1 unit and 2 (8\%) patients received 2 units. The total transfusion mean was 1.36 units (0-3). The postoperative mean hemoglobin value was $11.24 \mathrm{~g} \mathrm{dL}^{-1}$ (Table 2). All of the patients were followed up in the orthopedics ward, and none of them needed intensive care.

\section{DISCUSSION}

In this retrospective study, it was shown that low-dose TXA administered at a dose of $10 \mathrm{mg} / \mathrm{kg}$ loading, and $1 \mathrm{mg} / \mathrm{kg} / \mathrm{h}$ maintenance, in AIS patients who underwent PSF, decreased intraoperative bleeding and the need for transfusion.

In the literature, the use of TXA in heart, liver, trauma, and solid organ surgery is very common and has been shown to reduce bleeding and the need for transfusion ${ }^{(10,11)}$. Also, there is an increasing number of publications on its use in pediatric scoliosis surgery ${ }^{(12-14)}$. In a meta-analysis of 581 patients, including AIS patients, the usage of TXA was shown to reduce bleeding and the need for transfusion ${ }^{(15)}$. In another meta-analysis including 2500 AIS patients, TXA has been shown to reduce the duration of surgery without increasing complications, as well as reducing the estimated intraoperative blood loss and blood transfusion need $^{(16)}$.

In recent publications, it is seen that TXA is used in very different loading and maintenance doses in AIS surgeries ${ }^{(17-21)}$. The loading doses of 20,50 , and $100 \mathrm{mg} / \mathrm{kg}$ used in these studies are high. In our study, a low dose protocol was applied with a loading dose of $10 \mathrm{mg} / \mathrm{kg}$, and a maintenance dose of $1 \mathrm{mg} / \mathrm{kg}$. In our study, an average of 11 vertebrae were fused and the average EBL was $616 \mathrm{~mL}$. In a study where the mean vertebrae fusion numbers were similar to our study, but the dose of TXA used was 10 times higher (loading $100 \mathrm{mg} / \mathrm{kg}$ maintenance $10 \mathrm{mg} / \mathrm{kg}$ ), the EBL was found to be $619 \mathrm{~mL}$, similar to our study ${ }^{(18)}$. In another study in which a $50 \mathrm{mg} / \mathrm{kg}$ loading and $5 \mathrm{mg} / \mathrm{kg}$ maintenance dose was administered in AIS patients, an average of 10 levels were fused, and the EBL in this study was found to be $695 \mathrm{~mL}$, once again, similar to our study ${ }^{(17)}$. However, compared to our study, the amount of RBC units used intraoperatively was found to be lower in this study ( 0.8 vs 0.3 ). Our transfusion strategy may also play a role in explaining this difference. In our study, 9 patients (36\%) did not receive a transfusion in the intraoperative period, while 12 patients (48\%) received 1 unit and 4 patients (16\%) received 2 units of RBC transfusion. In all our scoliosis surgeries, we accepted the threshold hb level as $10 \mathrm{~g} \mathrm{dL}^{-1}$ to preserve the perfusion of the spinal cord. We performed transfusion at levels below these values, and also in cases of severe hemodynamic disturbances and hypotension, that could not be explained by other factors. This situation can be attributed to the differences 
in the transfusion protocol between clinics, and consequently the differences in the results of the studies can be explained. In a study in which $100 \mathrm{mg} / \mathrm{kg}$ loading and $10 \mathrm{mg} / \mathrm{kg} / \mathrm{h}$ maintenance doses of TXA were used in AIS surgery, it was reported that intraoperative blood loss was significantly decreased compared to the placebo group $(800 \mathrm{~mL}$ vs $1376 \mathrm{~mL}$ respectively). However, transfusion volumes were similar ${ }^{(19)}$. This is attributed by the authors to the lack of published standard criteria for blood transfusion thresholds, as in our study. In addition to hemoglobin and hemodynamic parameters, it was also emphasized that blood transfusion can be performed in order to be one step ahead of bleeding, depending on the previous experience of the anesthesiologists. Similarly, Sethna et al.(12), using TXA at a $100 \mathrm{mg} / \mathrm{kg}$ loading and $10 \mathrm{mg} / \mathrm{kg} / \mathrm{h}$ maintenance dose, found a $41 \%$ reduction in blood los, but reported that the amount of transfused blood was not different from the placebo group. There are two randomized controlled trials that applied and evaluated the low dose TXA regimen we used. One of these studies reported a 30\% reduction in the number of blood products transfused and no difference in blood loss ${ }^{(13)}$, while the other reported a significant reduction in intraoperative EBL and total drained blood, similar to our study ${ }^{(7)}$.

The recent studies, using very different and high dose schemes, have not been planned in accordance with the pharmacokinetics of TXA. Johnson et al. ${ }^{(17)}$ emphasized that guidelines should be created using pharmacokinetic data and modeling, to increase the effectiveness of TXA, reduce the side effects and find the minimum effective dose. Goobie and Faraoni. ${ }^{(22)}$ conducted a study about pharmacokinetic modeling and simulations for TXA. They recommended the loading dose as $10-30 \mathrm{mg} / \mathrm{kg}$, and maintenance as $5-10 \mathrm{mg} / \mathrm{kg} / \mathrm{h}$, for pediatric trauma and pediatric non-cardiac surgery. Apparently, in line with these recommendations, it has been revealed that very high doses were used in many recent studies.

Very high doses of TXA may be associated with serious undesirable side effects. Nausea, vomiting, diarrhea, deep vein thrombosis, pulmonary embolism, myocardial infarction, hypersensitivity reaction, renal failure, and seizures may be seen $^{(9,23,24)}$. A TXA-related seizure is an important side effect of TXA, and its incidence has been reported to be more frequent than originally thought. Seizures usually occur in the early postoperative period after surgery, and are due to the fast entrance of TXA into the central nervous system in high concentrations. The administration of the lowest effective loading dose to prevent seizures has emphasized the need for dose adjustment in patients with renal dysfunction, a history of seizures, or any other reason for impaired blood-brain barrier ${ }^{(25)}$. With the occurrence of seizures during recovery, it can be concluded that the effect of potential seizures, in studies with high doses, was attenuated or some of them were overlooked, due to the residual effect of anesthesia. In addition, cases with anaphylactic reactions related to TXA use have also been reported ${ }^{(26,27)}$. Although the number of patients in our study was small, we did not encounter any side effects related to the use of TXA at the doses we used. Surgical complications which developed in two patients were resolved by debridement and extension of the system to higher levels.

The retrospective nature of our study, the absence of a control group, and the small number of patients are important limitations. However, even with these limitations, it is important to note that we have seen low blood loss with low dose TXA protocol, in long segment fusion, which is close to high dose studies in the literature.

\section{CONCLUSION}

The use of low-dose TXA with minimal adverse effects would be a reliable method to reduce bleeding in AIS surgery until an effective pharmacokinetic study specific to the pediatric spinal surgery population has been performed, and a minimum effective dose is found in the future.

\section{Ethics}

Ethics Committee Approval: This retrospective study approved by Başkent University Instutional Review Board (KA21/29) and supported with materials by Başkent University Research Fund. Informed Consent: Retrospective study.

\section{Authorship Contributions}

Concept: Ç.B., Ü.Ö.G., Design: Ç.B., Ü.Ö.G., Data Collection or Processing: Ü.Ö.G., Analysis or Interpretation: Ç.B., Literature Search: Ç.B., Ü.Ö.G., Writing: Ç.B., Ü.Ö.G.

Conflict of Interest: The authors declare that they have no conflict of interest.

Financial Disclosure: The authors declared that this study received no financial support.

\section{REFERENCES}

1. Akcali O, Kosay C. Surgical reconstruction of deformed spine in adolescent idiopathic scoliosis. World Spinal Column J. 2011;2:62-7.

2. Cristante AF, Alvim Borges $\mathrm{P}$, Roberto Barbosa A, Biraghi Letaif O, Martus Marcon R, De Barros-Filho TEP. Predictive factors for perioperative blood transfusion in surgeries for correction of idiopathic, neuromuscular or congenital scoliosis. Clinics. 2014;69;672-6.

3. Shapiro F, Sethna N. Blood loss in pediatric spine surgery. Eur Spine J. 2004;13(Suppl 1):S6-17. doi: 10.1007/s00586-004-0760-y.

4. Schwarzkopf R, Chung C, Park JJ, Walsh M, Spivak JM, Steiger D. Effects of perioperative blood product use on surgical site infection following thoracic and lumbar spinal surgery. Spine (Phila Pa 1976). 2010;35:340-6.

5. Brookfield KF, Brown MD, Henriques SM, Buttacavoli FA, Seitz AP. Allogeneic transfusion after predonation of blood for elective spine surgery. Clin Orthop Relat Res. 2008;466:1949-53.

6. Copley LAB, Stephens Richards B, Safavi FZ, Newton PO. Hemodilution as a method to reduce transfusion requirements in adolescent spine fusion surgery. Spine (Phila Pa 1976). 1999;24:219-24.

7. Verma K, Errico T, Diefenbach C, Hoelscher C, Peters A, Dryer J, et al. The relative efficacy of antifibrinolytics in adolescent idiopathic scoliosis: a prospective randomized trial. J Bone Joint Surg Am. 2014;96:e80. doi: 10.2106/JBJS.L.00008. 
8. Bowen RE, Gardner S, Scaduto AA, Eagan M, Beckstead. Efficacy of intraoperative cell salvage systems in pediatric idiopathic scoliosis patients undergoing posterior spinal fusion with segmental spinal instrumentation. Spine (Phila Pa 1976). 2010;35:246-51.

9. Wang $M$, Zheng $X F$, Jiang LS. Efficacy and safety of antifibrinolytic agents in reducing perioperative blood loss and transfusion requirements in scoliosis surgery: A systematic review and metaanalysis. PLoS One. 2015;10:e0137886. doi: 10.1371/journal. pone.0137886.

10. Henry DA, Carless PA, Moxey AJ, O' Connell D, Stokes B], Fergusson DA, et al. Anti-fibrinolytic use for minimising perioperative allogeneic blood transfusion. Cochrane Database Syst Rev. 2011:CD001886. doi: 10.1002/14651858.CD001886.pub3.

11. Ortmann E, Besser MW, Klein AA. Antifibrinolytic agents in current anaesthetic practice. Br J Anaesth. 2013;111:549-63.

12. Sethna NF, Zurakowski D, Brustowicz RM, Bacsik J, Sullivan LJ, Shapiro F. Tranexamic acid reduces intraoperative blood loss in pediatric patients undergoing scoliosis surgery. Anesthesiology. 2005;102:727-32.

13. Neilipovitz DT, Murto K, Hall L, Barrowman NJ, Splinter WM. A randomized trial of tranexamic acid to reduce blood transfusion for scoliosis surgery. Anesth Analg. 2001;93:82-7.

14. Grant JA, Howard J, Luntley J, Harder J, Aleissa S, Parsons D. Perioperative blood transfusion requirements in pediatric scoliosis surgery: The efficacy of tranexamic acid. J Pediatr Orthop. 2009;29:300-4.

15. Yang B, Li H, Wang D, He X, Zhang C, Yang P. Systematic review and meta-analysis of perioperative intravenous tranexamic acid use in spinal surgery. PLoS One. 2013;8:e55436. doi: 10.1371/journal. pone.0055436.

16. Zhong J, Cao K, Wang B, Zhou X, Lin N, Lu H. The perioperative efficacy and safety of tranexamic acid in adolescent idiopathic scoliosis. World Neurosurg. 2019;129:e726-32.

17. Johnson DJ, Johnson CC, Goobie SM, Nami N, Wetzler JA, Sponseller PD, et al. High-dose Versus Low-dose Tranexamic Acid to Reduce
Transfusion Requirements in Pediatric Scoliosis Surgery. J Pediatr Orthop. 2017;37(8):e552-e7.

18. Sui WY, Ye F, Yang JL. Efficacy of tranexamic acid in reducing allogeneic blood products in adolescent idiopathic scoliosis surgery. BMC Musculoskelet Disord. 2016;17:1-5.

19. Lykissas MG, Crawford AH, Chan G, Aronson LA, Al-Sayyad MJ. The effect of tranexamic acid in blood loss and transfusion volume in adolescent idiopathic scoliosis surgery: A single-surgeon experience. I Child Orthop. 2013;7:245-9.

20. Yagi M, Hasegawa J, Nagoshi N, lizuka S, Kaneko S, Fukuda K, et al. Does the intraoperative tranexamic acid decrease operative blood loss during posterior spinal fusion for treatment of adolescent idiopathic scoliosis? Spine (Phila Pa 1976). 2012;37:16-20.

21. Shrestha IK, Ruan TY, Lin L, Tan M, Na XQ, Qu QC, et al. The efficacy and safety of high-dose tranexamic acid in adolescent idiopathic scoliosis: a meta-analysis. J Orthop Surg Res. 2021;16:53.

22. Goobie SM, Faraoni D. Tranexamic acid and perioperative bleeding in children: What do we still need to know? Curr Opin Anaesthesiol. 2019;32:343-52.

23. Cheriyan T, Maier SP, Bianco K, Slobodyanyuk K, Rattenni RN, Lafage $\mathrm{V}$, et al. Efficacy of tranexamic acid on surgical bleeding in spine surgery: A meta-analysis. Spine J. 2015;15:752-61.

24. Li ZJ, Fu X, Xing D, Zhang HF, Zang JC, Ma XL. Is tranexamic acid effective and safe in spinal surgery? A meta-analysis of randomized controlled trials. Eur Spine J. 2013;22:1950-7.

25. Lecker I, Wang DS, Whissell PD, Avramescu S, Mazer CD, Orser BA. Tranexamic acid-associated seizures: causes and treatment. Ann Neurol. 2016;79:18-26.

26. Chiem J, Ivanova I, Parker A, Krengel W, Jimenez N. Anaphylactic reaction to tranexamic acid in an adolescent undergoing posterior spinal fusion. Paediatr Anaesth. 2017;27:774-5.

27. Li PH, Trigg C, Rutkowski R, Rutkowski K. Anaphylaxis to tranexamic acid-a rare reaction to a common drug. J Allergy Clin Immunol Pract. 2017;5:839-41. 